\title{
A bound for the discrepancy of digital nets and its application to the analysis of certain pseudo-random number generators
}

\author{
by
}

\author{
Gerhard Larcher (Salzburg)
}

1. Introduction. The concept of digital nets is at the moment the most effective method for the construction of low-discrepancy point sets in the $s$-dimensional unit cube. Furthermore, by recent work it turned out that digital nets also play an important role in the analysis of certain pseudo-random number generators.

Until now the discrepancy of digital nets essentially was estimated by using discrepancy bounds valid for arbitrary nets. In this paper we give a more sensible - in some sense - discrepancy bound, especially for digital nets generated over a finite field of prime order, and we apply this bound for improving some results concerning the serial test of certain pseudo-random number generators.

The serial test is a test for the statistical independence of successive pseudo-random numbers. For a pseudo-random number sequence $x_{0}, x_{1}, \ldots$ $\ldots, x_{N-1}$ in $[0,1)$ and a fixed dimension $s \geq 2$ let the serial set $\left(\mathbf{x}_{n}\right)_{n \geq 0}$ of dimension $s$ be defined by $\mathbf{x}_{n}:=\left(x_{n}, x_{n+1}, \ldots, x_{n+s-1}\right) \in[0,1)^{s}$ for $n=0,1, \ldots, N-1$. (Here we consider the sequence $\left(x_{n}\right)_{n \geq 0}$ to be periodic with period $N$.) We then consider the usual star-discrepancy $D_{N}^{*}$ of this sequence in $[0,1)^{s}$. $D_{N}^{*}$ is defined by

$$
D_{N}^{*}=\sup _{B}\left|\frac{A_{N}(B)}{N}-\lambda(B)\right|,
$$

where the supremum is over all subintervals $B$ in $[0,1)^{s}$ with one vertex at the origin, $A_{N}(B)$ denotes the number of elements of the sequence belonging to $B$, and $\lambda(B)$ is the $s$-dimensional volume of $B$.

Small discrepancy guarantees good statistical independence properties of the successive elements of the pseudo-random sequence.

1991 Mathematics Subject Classification: 11K38, 11K45. 
K. F. Roth [11] has shown that for every dimension $s \geq 2$ there exists a constant $c_{s}>0$ such that for every $N \geq 2$ and each sequence $y_{0}, y_{1}, \ldots, y_{N-1}$ in $[0,1)^{s}$, for the corresponding star-discrepancy $D_{N}^{*}$ of the sequence we have

$$
D_{N}^{*} \geq c_{s} \frac{(\log N)^{(s-1) / 2}}{N} .
$$

It is a famous conjecture that this still holds if the exponent $(s-1) / 2$ of the logarithm is replaced by $s-1$. Until now this was only proved for the dimensions $s=1$ and $s=2$ (see [12]). So by "small discrepancy" we mean a discrepancy of an order $(\log N)^{A} / N$ with $A$ not much larger than $s-1$.

In this paper we consider three widely used pseudo-random number generation methods: the recursive matrix method (combined with the $p$-adic digit method), the digital multistep method, and the generalized feedback shift-register method. These methods have the property that their serial sets show in some sense a "net property" and even a "digital net property". For the theory of nets and for more details and a discussion concerning the serial test see the excellent monograph [4] of Niederreiter, and the various references given there.

For all these generation methods we show the existence of parameters which provide pseudo-random number sequences with large period and with an extremely small discrepancy for its serial sets. We thereby improve results which are given in, or can be deduced from, [6], [3] and [2].

Note that it is not the intention of this paper to discuss or to evaluate different pseudo-random number generation methods or to give comments on advantages and disadvantages of various pseudo-random number tests.

2. A discrepancy bound for digital nets. The concept of digital nets over a certain ring is at the moment the most effective method for the construction of low-discrepancy sequences in an $s$-dimensional unit cube. We just mention the powerful construction methods given by Niederreiter and Xing for example in [8]-[10] which are based on the digital construction concept over a finite field. In this section we recall the notion of digital nets and we give the new discrepancy bound in Proposition 1.

Let $p$ be a prime, let $F_{p}$ be the finite field of order $p$ and use the natural identification between the elements of the field and the digits between 0 and $p-1$.

For integers $s \geq 2, m \geq 2$ and $N=p^{m}$ the sequence $\mathbf{x}_{0}, \ldots, \mathbf{x}_{N-1} \in$ $[0,1)^{s}$ with $\mathbf{x}_{n}:=\left(x_{n}(1), \ldots, x_{n}(s)\right)$ is called a digital net over $F_{p}$ if there exist $s m \times m$ matrices $A_{1}, \ldots, A_{s}$ over $F_{p}$ such that for all $n=0, \ldots, N-1$ and $i=1, \ldots, s$ we have

$$
x_{n}(i)=\frac{1}{N} \tau\left(A_{i} \cdot \tau^{-1}(n)\right) .
$$


Here we denote by $\tau$ the following bijection between $F_{p}^{m}$ and $\left\{0, \ldots, p^{m}-1\right\}$ :

$$
\tau\left(\left(a_{0}, \ldots, a_{m-1}\right)\right):=a_{0}+a_{1} p+\ldots+a_{m-1} p^{m-1} .
$$

The quality of the distribution of a digital net of course essentially depends on the properties of the defining matrices $A_{i}$ (see for example Theorem 4.28 of [4]).

Let $A_{1}, \ldots, A_{s}$ be given and denote by $a_{j}^{(i)} \in F_{p}^{m}$ with $j=1, \ldots, m$ the rows of the matrix $A_{i}$ for $i=1, \ldots, s$. For $0 \leq w \leq s$, a $w$-tuple $\left(d_{1}, \ldots, d_{w}\right)$ of non-negative integers is called admissible with respect to $A_{1}, \ldots, A_{s}$ if the system $\left\{a_{j}^{(i)}: j=1, \ldots, d_{i}, i=1, \ldots, w\right\}$ is linearly independent over $F_{p}$. For $w=0$ we define the "zero-tuple" () to be admissible. For $w \leq s-1$ and $\left(d_{1}, \ldots, d_{w}\right)$ admissible we set $h\left(d_{1}, \ldots, d_{w}\right):=\max \{h \geq 0 \mid$ $\left(d_{1}, \ldots, d_{w}, h\right)$ is admissible $\}$.

Then we have:

Proposition 1. Let $D^{*}$ denote the star-discrepancy of the digital net $\mathbf{x}_{0}, \ldots, \mathbf{x}_{p^{m}-1}$ over $F_{p}$ defined by $A_{1}, \ldots, A_{s}$. Then

$$
D^{*} \leq \sum_{w=0}^{s-1}(p-1)^{w} \sum_{\substack{\left(d_{1}, \ldots, d_{w}\right) \text { admissible } \\ d_{i}>0}} p^{-\left(d_{1}+\ldots+d_{w}+h\left(d_{1}, \ldots, d_{w}\right)\right)} .
$$

Proof. By the definitions, if $\left(d_{1}, \ldots, d_{w}\right)$ is admissible and we let

$$
B \subseteq[0,1]^{s} \quad \text { with } \quad B=\prod_{i=1}^{w}\left[\frac{a_{i}}{p^{d_{i}}}, \frac{b_{i}}{p^{d_{i}}}\right) \times[0,1)^{s-w}
$$

with integers $0 \leq a_{i}<b_{i} \leq p^{d_{i}}$ (we call such an interval an admissible interval), then $B$ contains exactly

$$
p^{m-\left(d_{1}+\ldots+d_{w}\right)} \prod_{i=1}^{w}\left(b_{i}-a_{i}\right)
$$

of the net points.

Let $M=\prod_{i=1}^{s}\left[0, \alpha_{i}\right) \subseteq[0,1)^{s}$ with $\alpha_{i}:=\sum_{j=1}^{\infty} \alpha_{j}^{(i)} / p^{j}$ for $i=1, \ldots, s$ be taken arbitrarily. (If the representation of some $\alpha_{i}$ is not unique then we use an infinite representation.) Then on the one hand we have

$$
\widetilde{M}:=\bigcup_{\substack{\left(d_{1}, \ldots, d_{s}\right) \text { admissible } \\ d_{i}>0}} \prod_{i=1}^{s}\left[\sum_{j=1}^{d_{i}-1} \frac{\alpha_{j}^{(i)}}{p^{j}}, \sum_{j=1}^{d_{i}} \frac{\alpha_{j}^{(i)}}{p^{j}}\right) \subseteq M .
$$

The intervals in the above union are pairwise disjoint and admissible. On 
the other hand, we will show by induction on $s$ that

$$
\begin{aligned}
M \subseteq & \widetilde{M} \cup \bigcup_{w=0}^{s-1} \bigcup_{\substack{\left(d_{1}, \ldots, d_{w}\right) \text { admissible } \\
d_{i}>0}}\left(\prod_{i=1}^{w}\left[\sum_{j=1}^{d_{i}-1} \frac{\alpha_{j}^{(i)}}{p^{j}}, \sum_{j=1}^{d_{i}} \frac{\alpha_{j}^{(i)}}{p^{j}}\right)\right. \\
& \times\left[\sum_{j=1}^{h\left(d_{1}, \ldots, d_{w}\right)} \frac{\alpha_{j}^{(w+1)}}{p^{j}}, \sum_{j=1}^{h\left(d_{1}, \ldots, d_{w}\right)} \frac{\alpha_{j}^{(w+1)}}{p^{j}}+\frac{1}{p^{h\left(d_{1}, \ldots, d_{w}\right)}}\right) \\
& \left.\times[0,1)^{s-w-1}\right) .
\end{aligned}
$$

(Again all intervals in the second union above are admissible.) For $s=1$ the right hand side above is

$$
\begin{aligned}
\bigcup_{d_{1} \text { admissible }}\left[\sum_{j=1}^{d_{1}-1} \frac{\alpha_{j}^{(1)}}{p^{j}}, \sum_{j=1}^{d_{1}} \frac{\alpha_{j}^{(1)}}{p^{j}}\right) \cup\left[\sum_{j=1}^{h()} \frac{\alpha_{j}^{(1)}}{p^{j}}, \sum_{j=1}^{h()} \frac{\alpha_{j}^{(1)}}{p^{j}}+\frac{1}{p^{h()}}\right) \\
=\left[0, \sum_{j=1}^{h()} \frac{\alpha_{j}^{(1)}}{p^{j}}+\frac{1}{p^{h()}}\right),
\end{aligned}
$$

which contains $M=\left[0, \alpha_{1}\right)$. Assume the assertion is true up to dimension $s-1$ and consider

$$
M=\prod_{i=1}^{s-1}\left[0, \alpha_{i}\right) \times\left[0, \alpha_{s}\right) .
$$

By induction,

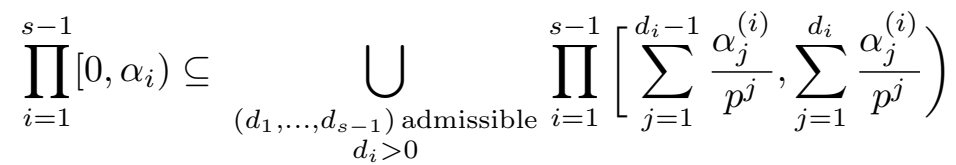

$$
\begin{aligned}
& \cup \bigcup_{w=0}^{s-2} \bigcup_{\substack{\left(d_{1}, \ldots, d_{w}\right) \text { admissible } \\
d_{i}>0}}\left(\prod_{i=1}^{w}\left[\sum_{j=1}^{d_{i}-1} \frac{\alpha_{j}^{(i)}}{p^{j}}, \sum_{j=1}^{d_{i}} \frac{\alpha_{j}^{(i)}}{p^{j}}\right)\right. \\
& \times\left[\sum_{j=1}^{h\left(d_{1}, \ldots, d_{w}\right)} \frac{\alpha_{j}^{(w+1)}}{p^{j}}, \sum_{j=1}^{h\left(d_{1}, \ldots, d_{w}\right)} \frac{\alpha_{j}^{(w+1)}}{p^{j}}+\frac{1}{p^{h\left(d_{1}, \ldots, d_{w}\right)}}\right) \\
& \left.\times[0,1)^{s-w-2}\right) .
\end{aligned}
$$

We extend each of the $(s-1)$-dimensional intervals $J$ on the right hand side above to an $s$-dimensional interval $J^{\prime}$ such that $M$ is contained in the union of these extensions. 
If $J$ is part of the first big union above, that is, if it is of the form

$$
\prod_{i=1}^{s-1}\left[\sum_{j=1}^{d_{i}-1} \frac{\alpha_{j}^{(i)}}{p^{j}}, \sum_{j=1}^{d_{i}} \frac{\alpha_{j}^{(i)}}{p^{j}}\right)
$$

for some admissible $\left(d_{1}, \ldots, d_{s-1}\right)$, then we take

$$
\begin{aligned}
J^{\prime}:= & \prod_{i=1}^{s-1}\left[\sum_{j=1}^{d_{i}-1} \frac{\alpha_{j}^{(i)}}{p^{j}}, \sum_{j=1}^{d_{i}} \frac{\alpha_{j}^{(i)}}{p^{j}}\right) \\
& \times\left(\bigcup_{k=1}^{h\left(d_{1}, \ldots, d_{s-1}\right)}\left[\sum_{j=1}^{k-1} \frac{\alpha_{j}^{(s)}}{p^{j}}, \sum_{j=1}^{k} \frac{\alpha_{j}^{(s)}}{p^{j}}\right)\right. \\
& \left.\cup\left[\sum_{j=1}^{h\left(d_{1}, \ldots, d_{s-1}\right)} \frac{\alpha_{j}^{(s)}}{p_{j}}, \sum_{j=1}^{h\left(d_{1}, \ldots, d_{s-1}\right)} \frac{\alpha_{j}^{(s)}}{p^{j}}+\frac{1}{p^{h\left(d_{1}, \ldots, d_{s-1}\right)}}\right)\right) .
\end{aligned}
$$

If $J$ is part of the second big union then we just extend by $[0,1)$.

By inserting we obtain

$$
\begin{aligned}
M \subseteq & \bigcup_{\substack{\left(d_{1}, \ldots, d_{s-1}\right) \text { admissible } \\
d_{i}>0}}\left(\prod_{i=1}^{s-1}\left[\sum_{j=1}^{d_{i}-1} \frac{\alpha_{j}^{(i)}}{p^{j}}, \sum_{j=1}^{d_{i}} \frac{\alpha_{j}^{(i)}}{p^{j}}\right)\right. \\
& \left.\times \bigcup_{k=1}^{h\left(d_{1}, \ldots, d_{s-1}\right)}\left[\sum_{j=1}^{k-1} \frac{\alpha_{j}^{(s)}}{p^{j}}, \sum_{j=1}^{k} \frac{\alpha_{j}^{(s)}}{p^{j}}\right)\right) \\
& \cup \bigcup_{\left(d_{1}, \ldots, d_{s-1}\right) \text { admissible }}\left(\prod_{i=1}^{s-1}\left[\sum_{j=1}^{d_{i}-1} \frac{\alpha_{j}^{(i)}}{p^{j}}, \sum_{j=1}^{d_{i}} \frac{\alpha_{j}^{(i)}}{p^{j}}\right)\right. \\
& \left.\times\left[\sum_{j=1}^{h\left(d_{1}, \ldots, d_{s-1}\right)} \frac{\alpha_{j}^{(s)}}{p^{j}}, \sum_{j=1}^{h\left(d_{1}, \ldots, d_{s-1}\right)} \frac{\alpha_{j}^{(s)}}{p^{j}}+\frac{1}{p^{h\left(d_{1}, \ldots, d_{s-1}\right)}}\right)\right) \\
& \cup \bigcup_{w=0}^{s-2}\left(\prod_{i=1}^{w}\left[\sum_{j=1}^{d_{i}-1} \frac{\alpha_{j}^{(i)}}{p^{j}}, \sum_{j=1}^{d_{i}} \frac{\alpha_{j}^{(i)}}{p^{j}}\right)\right. \\
& \times\left[\sum_{j=1}^{h\left(d_{1}, \ldots, d_{w}\right) \text { admissible }} d_{d_{i}>0} \frac{\alpha_{j}^{(w+1)}}{p^{j}}, \sum_{j=1}^{h\left(d_{1}, \ldots, d_{w}\right)} \frac{\alpha_{j}^{(w+1)}}{p^{j}}+\frac{1}{p^{h\left(d_{1}, \ldots, d_{w}\right)}}\right) \\
& \left.\times[0,1)^{s-w-1}\right),
\end{aligned}
$$

and the induction is finished. 
So we obtain

$$
\left|\frac{A_{N}(M)}{N}-\lambda(M)\right| \leq \sum_{w=0}^{s-1}(p-1)^{w} \sum_{\substack{\left(d_{1}, \ldots, d_{w}\right) \\ \text { admissible } \\ d_{i}>0}} p^{-\left(d_{1}+\ldots+d_{w}+h\left(d_{1}, \ldots, d_{w}\right)\right)}
$$

and the result follows.

3. The recursive matrix method. The recursive matrix method was introduced in full generality by Niederreiter in [5], and it was studied in detail for example in [6] and [7]. Here we only consider the case of recursive matrix methods of order one. This is a combination of the classical matrix method for the generation of pseudo-random vectors (see [4]), combined with a $p$-adic digit method.

The method is the following. Let $p$ be a prime and let $F_{p}$ be again the finite field of order $p$. Let $m$ be a positive integer and let $A$ be a non-singular $m \times m$ matrix over $F_{p}$. A sequence $\mathbf{z}_{0}, \mathbf{z}_{1}, \ldots$ of row vectors from $F_{p}^{m}$ is generated by choosing an initial vector $\mathbf{z}_{0}$ different from $\mathbf{0}$ and by

$$
\mathbf{z}_{n+1}:=\mathbf{z}_{n} \cdot A \quad \text { for } n=0,1, \ldots
$$

We now derive pseudo-random numbers $x_{n}$ in $[0,1)$ from $\mathbf{z}_{n}:=$ $\left(z_{n}^{(1)}, \ldots, z_{n}^{(m)}\right) \in F_{p}^{m}$ in the following way. We identify the elements $z \in F_{p}$ in the natural way with digits $z \in\{0, \ldots, p-1\}$. Then

$$
x_{n}:=\sum_{j=1}^{m} z_{n}^{(j)} p^{-j} \quad \text { for } n=0,1, \ldots
$$

The sequence $\left(\mathbf{z}_{n}\right)_{n \geq 0}$ and therefore $\left(x_{n}\right)_{n \geq 0}$ is purely periodic because of the non-singularity of the matrix $A$, with (least) period at most $p^{m}-1$. This maximal (least) period is attained if and only if the polynomial $\operatorname{det}\left(x \cdot I_{m}-A\right)$ of degree $m$ is a primitive polynomial over $F_{p}$. (Here $I_{m}$ is the $m \times m$ identity matrix.) This is shown for example in Theorem 10.2 of [4]. In the following we restrict ourselves to this, for practical purposes most important, case of maximal period.

Let in the following $q:=p^{m}$. In Theorem 2 of [6] it was shown that a sequence $\left(\mathbf{z}_{n}\right)_{n \geq 0}$ with $\mathbf{z}_{n}:=\left(z_{n}^{(1)}, \ldots, z_{n}^{(m)}\right) \in F_{p}^{m}$ is a recursive vector sequence of the above form of period $T:=p^{m}-1$ if and only if there is a primitive element $\sigma$ of $F_{q}$ and a basis $\beta_{1}, \ldots, \beta_{m}$ of $F_{q}$ over $F_{p}$ such that $z_{n}^{(j)}=\operatorname{Tr}\left(\beta_{j} \sigma^{n}\right)$ for $1 \leq j \leq m$ and $n \geq 0$. Here $\operatorname{Tr}$ is the trace function from $F_{q}$ to $F_{p}$.

Concerning the star-discrepancy $D_{T}^{*(s)}$ of the serial sets of dimension $s$ of these sequences, the following was shown in [6]. 
Let $2 \leq s \leq m$ and let $\sigma$ be a fixed primitive element of $F_{q}$. Then for $D_{T}^{*(s)}$ we have on the average

$$
D_{T}^{*(s)} \leq c(s) \frac{(\log T)^{s}}{T}
$$

with an implied constant depending only on $s$, where the average is taken over all ordered bases of $F_{q}$ over $F_{p}$.

From this we at once deduce the following. Let $2 \leq s \leq m$, let $\sigma$ be a fixed primitive element of $F_{q}$ and let $\mathcal{B}$ be the set of ordered bases of $F_{q}$ over $F_{p}$. Let $0<\gamma<1$ be given. Then the number of bases $B \in \mathcal{B}$ for which for the discrepancy $D_{T}^{*(s)}(B)$ of the $s$-dimensional serial set of the corresponding sequence we have

$$
D_{T}^{*(s)}(B) \leq \frac{1}{1-\gamma} c(s) \frac{(\log T)^{s}}{T}
$$

is at least $\gamma|\mathcal{B}|$.

We improve this result (at least for small $p$ ) by almost one logarithmic factor in the following way:

Theorem 1. Let $2 \leq s \leq m$, let $\sigma$ be a primitive element of $F_{q}$ and let $\mathcal{B}$ be the set of ordered bases of $F_{q}$ over $F_{p}$. Let $0<\gamma<1$ be given. Then the number of bases $B \in \mathcal{B}$ for which for the discrepancy $D_{T}^{*(s)}(B)$ of the $s$-dimensional serial set $\mathbf{x}_{0}, \ldots, \mathbf{x}_{T-1}$ of the corresponding sequence we have

$$
\begin{aligned}
D_{T}^{*(s)}(B) \leq & \frac{1}{T}+\frac{1}{p^{m}} \sum_{w=0}^{s-1}(p-1)^{w}\left(\begin{array}{c}
m \\
w
\end{array}\right) \\
& \times\left[(s-1)\left(\frac{p}{p-1}\right)^{2} \frac{2}{1-\gamma} p \log m\right. \\
& \left.+\left(\frac{p}{p-1}\right)^{2} \frac{2}{1-\gamma}\left(1+{ }_{p} \log \frac{4}{1-\gamma}\right)+\frac{1+\gamma}{1-\gamma}\right] \\
= & \mathcal{O}\left(\frac{(\log T)^{s-1} \log \log T}{T}\right)
\end{aligned}
$$

is at least $\gamma|\mathcal{B}|$. (Here we denote by ${ }_{p} \log$ the logarithm to base $\left.p.\right)$

Re mark 1. Note that the constant in the $\mathcal{O}$-result of Theorem 1 does also depend on $p$.

Remark 2. For example, in the case $p=2$ for at least half the bases $B$ in $\mathcal{B}$, we have

$$
D_{T}^{*(s)}(B) \leq 68 \frac{1}{2^{m}} \sum_{w=0}^{s-1}\left(\begin{array}{l}
m \\
w
\end{array}\right)+16(s-1) \frac{2 \log m}{2^{m}} \sum_{w=0}^{s-1}\left(\begin{array}{l}
m \\
w
\end{array}\right) .
$$


Remark 3. The above discrepancy estimates coincide up to the $\log \log T$ factors with the conjectured general lower bound for the discrepancy of point sets in $[0,1)^{s}$.

Proof of Theorem 1. Let the recursive matrix sequence $x_{0}, \ldots$ $\ldots, x_{T-1}$ be defined by the primitive element $\sigma$ of $F_{q}$ and by the ordered basis $B=\left\{\beta_{1}, \ldots, \beta_{m}\right\}$ of $F_{q}$ over $F_{p}$. The $\beta_{i}$ are viewed as vectors of $F_{q}$ over $F_{p}$. By Theorem 5 of [6], the set $\mathbf{0}, \mathbf{x}_{0}, \mathbf{x}_{1}, \ldots, \mathbf{x}_{T-1}$ forms a digital net over $F_{p}$ which is generated by certain matrices, say $C_{1}, \ldots, C_{s}$. Let $c_{j}^{(i)} \in F_{p}^{m}$ for $1 \leq j \leq m$ be rows of $C_{i}$ for $1 \leq i \leq s$.

It is shown in the proof of that Theorem 5 that these $C_{1}, \ldots, C_{s}$ have the following property: for any non-negative integers $d_{i} \leq m, i=1, \ldots, s$, the system of vectors $\left\{c_{j}^{(i)}: 1 \leq j \leq d_{i}, 1 \leq i \leq s\right\}$ is linearly dependent over $F_{p}$ if and only if the system $\left\{\beta_{j} \sigma^{i-1}: 1 \leq j \leq d_{i}, 1 \leq i \leq s\right\}$ is. In the following we consider admissible $w$-tuples of integers with respect to the matrices $A_{i}(B)$ with rows $\beta_{j} \sigma^{i-1}, j=1, \ldots, m$, for $i=1, \ldots, s$ and we call them (for fixed $\sigma$ ) admissible for $B$. Then by Proposition 1 for the star-discrepancy $D_{T}^{*(s)}(B)$ of the set $\mathbf{0}, \mathbf{x}_{0}, \mathbf{x}_{1}, \ldots, \mathbf{x}_{T-1}$ we have

$$
D_{T}^{*(s)}(B) \leq \sum_{w=0}^{s-1}(p-1)^{w} \sum_{\begin{array}{c}
\left(d_{1}, \ldots, d_{w}\right) \\
\text { admissible for } B \\
d_{i}>0
\end{array}} p^{-\left(d_{1}+\ldots+d_{w}+h\left(d_{1}, \ldots, d_{w}\right)\right)} .
$$

For a non-negative integer $c$ let $\mathcal{M}(c)$ be the set of $B \in \mathcal{B}$ such that there exist positive integers $d_{1}, \ldots, d_{s}$ with $d_{1}+\ldots+d_{s}=m-c$ and with $\beta_{j} \sigma^{i-1}, j=1, \ldots, d_{i}, i=1, \ldots, s$, linearly dependent over $F_{p}$. We have

$$
|\mathcal{M}(c)| \leq \sum_{\begin{array}{c}
\mathbf{d}:=\left(d_{1}, \ldots, d_{s}\right) \\
d_{1}+\ldots+d_{s}=m-c \\
d_{i}>0
\end{array}} \sum_{\substack{\lambda:=\left(\lambda_{1}, \ldots, \lambda_{m-c}\right) \in \\
F_{p}^{m-c} \backslash\{\mathbf{0}\}}}|\mathcal{M}(\lambda, \mathbf{d})|
$$

with

$$
\begin{aligned}
\mathcal{M}(\lambda, \mathbf{d}):=\{B \in \mathcal{B} \mid & \lambda_{1} \beta_{1} \sigma^{0}+\ldots+\lambda_{d_{1}} \beta_{d_{1}} \sigma^{0}+\ldots \\
& \left.\ldots+\lambda_{d_{1}+\ldots+d_{s-1}+1} \beta_{1} \sigma^{s-1}+\ldots+\lambda_{m-c} \beta_{d_{s}} \sigma^{s-1}=0\right\} .
\end{aligned}
$$

We estimate the number of elements of $\mathcal{M}(\lambda, \mathbf{d})$. There is an $i \in\{1, \ldots, m-c\}$ with $\lambda_{i} \neq 0$. Without loss of generality assume $\lambda_{1} \neq 0$. Since $s \leq m$ and since $\sigma$ is primitive, we have $\lambda_{1} \sigma^{0}+\ldots+\lambda_{d_{1}+\ldots+d_{s-1}+1} \sigma^{s-1}$ $\neq 0$. So for arbitrarily chosen linearly independent $\beta_{2}, \ldots, \beta_{m}$ (there are $\left(p^{m}-1\right) \ldots\left(p^{m}-p^{m-2}\right)$ such choices $)$ there is at most one $\beta_{1}$ such that $\left(\beta_{1}, \ldots, \beta_{m}\right) \in \mathcal{B}$. Consequently,

$$
|\mathcal{M}(\lambda, \mathbf{d})| \leq\left(p^{m}-1\right)\left(p^{m}-p\right) \ldots\left(p^{m}-p^{m-2}\right)=|\mathcal{B}| \frac{1}{p^{m}-p^{m-1}}
$$


and therefore

$$
|\mathcal{M}(c)| \leq|\mathcal{B}| \frac{1}{p^{c}} \cdot \frac{p}{p-1}\left(\begin{array}{c}
m-c-1 \\
s-1
\end{array}\right) .
$$

Let $\overline{\mathcal{M}}(c):=\mathcal{B} \backslash \mathcal{M}(c)$. Then

$$
|\overline{\mathcal{M}}(c)| \geq|\mathcal{B}|(1-R(c)) \quad \text { with } \quad R(c):=\frac{1}{p^{c}} \cdot \frac{p}{p-1}\left(\begin{array}{c}
m-c-1 \\
s-1
\end{array}\right) .
$$

For a positive integer $c$ we now consider

$$
\begin{aligned}
\sum:= & \frac{1}{|\overline{\mathcal{M}}(c)|} \sum_{B \in \overline{\mathcal{M}}(c)} D_{T}^{*(s)}(B) \\
\leq & \frac{1}{|\overline{\mathcal{M}}(c)|} \sum_{B \in \overline{\mathcal{M}}(c)} \sum_{w=0}^{s-1}(p-1)^{w} \sum_{\begin{array}{c}
d_{1}, \ldots ., d_{w} \\
\text { admissible for } B \\
d_{i}>0
\end{array}} p^{-\left(d_{1}+\ldots+d_{w}+h\left(d_{1}, \ldots, d_{w}\right)\right)} \\
\leq & \frac{1}{|\overline{\mathcal{M}}(c)|} \sum_{w=0}^{s-1}(p-1)^{w} \sum_{B \in \overline{\mathcal{M}}(c)} \sum_{\begin{array}{c}
d_{1}, \ldots, d_{w} \\
\text { admissible for } B \\
d_{i}>0
\end{array}} p^{-\left(d_{1}+\ldots+d_{w}\right)} \\
& \times\left(\left(\sum_{i=m-\left(d_{1}+\ldots+d_{w}\right)-c+1}^{m} \sum_{\lambda}^{*} \frac{p}{p-1} \cdot \frac{1}{p_{i}}\right)+\frac{1}{p^{m-\left(d_{1}+\ldots+d_{w}\right)}}\right) .
\end{aligned}
$$

Here $\sum_{\lambda}^{*}$ means summation over all

$$
\lambda:=\left(\lambda_{1}, \ldots, \lambda_{d_{1}+\ldots+d_{w}+i}\right) \in F_{p}^{d_{1}+\ldots+d_{w}+i} \backslash\{\mathbf{0}\}
$$

for which

$$
\begin{aligned}
\lambda_{1} \beta_{1}+\ldots+\lambda_{d_{1}} \beta_{d_{1}}+\ldots+ & \lambda_{d_{1}+\ldots+d_{w-1}+1} \beta_{1} \sigma^{w-1}+\ldots+\lambda_{d_{1}+\ldots+d_{w}} \beta_{d_{w}} \sigma^{w-1} \\
& +\lambda_{d_{1}+\ldots+d_{w}+1} \beta_{1} \sigma^{w}+\ldots+\lambda_{d_{1}+\ldots+d_{w}+i} \beta_{i} \sigma^{w}=0 .
\end{aligned}
$$

The summand $1 / p^{m-\left(d_{1}+\ldots+d_{w}\right)}$ comes from the case where $h\left(d_{1}, \ldots, d_{w}\right)=$ $m-\left(d_{1}+\ldots+d_{w}\right)$ and the factor $p /(p-1)$ comes from the fact that whenever for given $w, B,\left(d_{1}, \ldots, d_{w}\right)$ and $i$ there is a possible summand $\lambda$ then there are at least $p-1$ such $\lambda$. 
Therefore

$$
\begin{aligned}
\sum \leq & \frac{1}{p^{m}} \sum_{w=0}^{s-1}(p-1)^{w}\left(\begin{array}{c}
m \\
w
\end{array}\right) \\
& +\frac{1}{|\overline{\mathcal{M}}(c)|} \cdot \frac{p}{p-1} \sum_{w=0}^{s-1}(p-1)^{w} \sum_{\substack{d_{1}, \ldots, d_{w}>0 \\
d_{1}+\ldots+d_{w} \leq m}} p^{-\left(d_{1}+\ldots+d_{w}\right)} \\
& \times \sum_{i=\max \left(0, m-\left(d_{1}+\ldots+d_{w}\right)-c+1\right)} \frac{1}{p^{i}} \sum_{\lambda \in F_{p}^{d_{1}+\ldots+d_{w}+i} \backslash\{\mathbf{0}\}}|\mathcal{M}(\lambda, \mathbf{d}, w)|,
\end{aligned}
$$

where $\mathcal{M}(\lambda, \mathbf{d}, w)$ is defined like $\mathcal{M}(\lambda, \mathbf{d})$ above but with $w$ instead of $s-1$. Estimating $|\mathcal{M}(\lambda, \mathbf{d}, w)|$ in the same way as $|\mathcal{M}(\lambda, \mathbf{d})|$ above, we obtain $|\mathcal{M}(\lambda, \mathbf{d}, w)| \leq|\mathcal{B}| /\left(p^{m}-p^{m-1}\right)$, and

$$
\begin{aligned}
\sum \leq & \frac{1}{p^{m}} \sum_{w=0}^{s-1}(p-1)^{w}\left(\begin{array}{l}
m \\
w
\end{array}\right) \\
& +\frac{1}{|\overline{\mathcal{M}}(c)|} \cdot \frac{p}{p-1} \cdot c \cdot \frac{|\mathcal{B}|}{p^{m}-p^{m-1}} \sum_{w=0}^{s-1}(p-1)^{w}\left(\begin{array}{c}
m \\
w
\end{array}\right) \\
= & \frac{1}{p^{m}} \sum_{w=0}^{s-1}(p-1)^{w}\left(\begin{array}{c}
m \\
w
\end{array}\right)\left[1+\left(\frac{p}{p-1}\right)^{2} c \frac{|\mathcal{B}|}{|\overline{\mathcal{M}}(c)|}\right]=: A(c) .
\end{aligned}
$$

Therefore for $\Gamma \geq 1$ the number of $B \in \mathcal{B}$ with $D_{T}^{*(s)}(B) \leq \Gamma A(c)$ is at least $(1-1 / \Gamma)(1-R(c))|\mathcal{B}|$.

Let now $\Gamma=(1+\gamma) /(1-\gamma)$ and choose $c \geq 1$ such that $R(c) \leq(1-\gamma) / 2$, that is,

which is satisfied for

$$
\frac{1}{p^{c}} \cdot \frac{p}{p-1}\left(\begin{array}{c}
m-c-1 \\
s-1
\end{array}\right) \leq \frac{1-\gamma}{2}
$$

$$
c \geq\left\lceil{ }_{p} \log \left(\frac{2 p}{(1-\gamma)(p-1)} m^{s-1}\right)\right\rceil
$$

(here $\lceil x\rceil$ means the smallest integer larger than or equal to $x$ ). By inserting the choices for $c$ and $\Gamma$ and by noting that the discrepancies of the point sets $\mathbf{x}_{0}, \ldots, \mathbf{x}_{T-1}$ and $\mathbf{0}, \mathbf{x}_{0}, \ldots, \mathbf{x}_{T-1}$ differ by at most $1 / T$, we obtain the result.

4. Shift-register sequences. In this section we consider both the digital multistep method and the generalized feedback shift-register (GFSR) method. For details see again [4], especially Chapter 9. 
(a) The digital multistep method. This method was introduced by Tausworthe in [13]. Let $p$ be a prime, let $k \geq 2$ be an integer and generate a $k$ th order linear recurring sequence $y_{0}, y_{1}, \ldots \in F_{p}$ by

$$
y_{n+k} \equiv \sum_{l=0}^{k-1} a_{l} y_{n+l}(\bmod p) \quad \text { for } n=0,1, \ldots
$$

where $y_{0}, \ldots, y_{k-1}$ are initial values not all zero, and where the coefficients $a_{0}, \ldots, a_{k-1} \in F_{p}$ are chosen in such a way that the characteristic polynomial $f(x):=x^{k}-\sum_{l=0}^{k-1} a_{l} x^{l} \in F_{p}[x]$ is a primitive polynomial over $F_{p}$. We then have a maximal possible period of length $p^{k}-1$ for the sequence $\left(y_{n}\right)_{n \geq 0}$.

In the digital multistep method we construct a pseudo-random number sequence $x_{0}, x_{1}, \ldots$ in $[0,1)$ by choosing an integer $m$ with $2 \leq m \leq k$ and by putting

$$
x_{n}:=\sum_{j=1}^{m} y_{m n+j} p^{-j} \quad \text { for } n=0,1, \ldots
$$

This sequence has a period $\left(p^{k}-1\right) /\left(m, p^{k}-1\right)$. (See [4], Lemma 9.1.) For various reasons it is most convenient to choose $m=k$ and to choose $k$ such that $\left(k, p^{k}-1\right)=1$. For given $k$ and $m$ the sequences $\left(x_{n}\right)_{n \geq 0}$ are uniquely determined by the primitive polynomial $f$ and by the initial values $y_{0}, \ldots, y_{k-1}$. Concerning the star-discrepancy $D_{T}^{*(s)}(f)$ of the $s$-dimensional serial set $\mathbf{x}_{n}:=\left(x_{n}, \ldots, x_{n+s+1}\right), n=0, \ldots, T-1$, it was shown in [3] that for $m=k$ and $\left(k, p^{k}-1\right)=1$ (and therefore $T=p^{k}-1$ ), and initial values $y_{0}, \ldots, y_{k-1}$ not all zero, we have, on the average,

$$
D_{T}^{*(s)}(f) \leq c(s, p) \frac{(\log T)^{s+1} \log \log T}{T}
$$

with an implied constant depending only on $p$ and $s$, where the average is taken over all primitive polynomials $f$ over $F_{p}$ of degree $k$. From this for arbitrary $\gamma, 0<\gamma<1$, we again immediately get the following. Let $\mathcal{Q}$ be the set of primitive polynomials $f$ over $F_{p}$ of degree $k$. Then the number of $f \in \mathcal{Q}$ for which the discrepancy $D_{T}^{*(s)}(f)$ of the $s$-dimensional serial set of the corresponding sequence satisfies

$$
D_{T}^{*(s)}(f) \leq \frac{1}{1-\gamma} c(s, p) \frac{(\log T)^{s+1} \log \log T}{T}
$$

is at least $\gamma|\mathcal{Q}|$.

We improve this result in the following:

Theorem 2. For a prime $p$ let $s \geq 2, m=k$ and $T:=p^{k}-1$ with $(k, T)=1$ and $y_{0}, \ldots, y_{k-1}$ in $F_{p}$, not all zero, be given. For fixed $\gamma, 0<$ $\gamma<1$, the number of $f \in \mathcal{Q}$ for which the star-discrepancy $D_{T}^{*(s)}(f)$ of the 
s-dimensional serial set of the corresponding digital multistep shift-register sequence defined by $f$ and the initial values $y_{0}, \ldots, y_{k-1}$ satisfies

$$
\begin{aligned}
D_{T}^{*(s)}(f) \leq & \frac{1}{T}+\frac{1}{p^{k}} \sum_{w=0}^{s-1}(p-1)^{w}\left(\begin{array}{c}
k \\
w
\end{array}\right) \\
& \times\left[s(s-1) \frac{p}{p-1} \cdot \frac{2}{1-\gamma} k \frac{p^{k}}{\phi(T)} p \log \left(k \frac{p^{k}}{\phi(T)}\right)\right. \\
& \left.+(s-1) \frac{p}{p-1} \cdot \frac{2}{1-\gamma} k \frac{p^{k}}{\phi(T)}\left(1+{ }_{p} \log \frac{2(s-1)}{1-\gamma}\right)+\frac{1+\gamma}{1-\gamma}\right] \\
= & \mathcal{O}\left(\frac{(\log T)^{s}(\log \log T)^{2}}{T}\right)
\end{aligned}
$$

is at least $\gamma|\mathcal{Q}|$. (Here $\phi$ is Euler's totient function.)

Proof. The proof runs along the same lines as the proof of Theorem 1. So it suffices to give the following details.

By Theorem 9.5 of [4], the $p^{k}$ points $\mathbf{0}, \mathbf{x}_{0}, \ldots, \mathbf{x}_{T-1}$ form a digital net over $F_{p}$ defined by $s$ matrices $C_{1}, \ldots, C_{s}$ with rows $c_{j}^{i} \in F_{p}^{k}$ with $1 \leq j \leq k$ for $1 \leq i \leq s$ with the following property: for non-negative integers $d_{i} \leq$ $k, i=1, \ldots, s$, the system of vectors $\left\{c_{j}^{i}: 1 \leq j \leq d_{i}, 1 \leq i \leq s\right\}$ is linearly dependent over $F_{p}$ if and only if the system $\left\{\alpha^{(i-1) k+j-1}: 1 \leq j \leq\right.$ $\left.d_{i}, 1 \leq i \leq s\right\}$ is. Here $\alpha$ is a root of $f$ in $F_{p^{k}}$, viewed as an element of the vector space $F_{p^{k}}$ over $F_{p}$. In the following we consider admissible $w$-tuples of integers with respect to the matrices $A_{i}(f)$ with rows $\alpha^{(i-1) k+j}, j=$ $0, \ldots, k-1$, for $i=1, \ldots, s$. For a non-negative integer $c$, for an $s$-tuple of non-negative integers $\mathbf{d}:=\left(d_{1}, \ldots, d_{s}\right)$ with $d_{1}+\ldots+d_{s}=k-c$ and $\lambda:=\left(\lambda_{1}, \ldots, \lambda_{k-c}\right) \in F_{p}^{k-c} \backslash\{\mathbf{0}\}$ let $\mathcal{M}(c, \lambda, \mathbf{d})$ be the set of $f \in \mathcal{Q}$ satisfying

$$
\begin{aligned}
\lambda_{1} \alpha^{0}+\ldots+\lambda_{d_{1}} \alpha^{d_{1}-1}+\lambda_{d_{1}+1} \alpha^{k}+\ldots+\lambda_{d_{1}+d_{2}} \alpha^{k+d_{2}-1}+\ldots \\
\ldots+\lambda_{d_{1}+\ldots+d_{s-1}+1} \alpha^{(s-1) k}+\ldots+\lambda_{d_{1}+\ldots+d_{s}} \alpha^{(s-1) k+d_{s}-1}=0 .
\end{aligned}
$$

Then

$$
|\mathcal{M}(c, \lambda, \mathbf{d})| \leq\left[\frac{(s-1) k+k-1}{k}\right]=s-1 .
$$

This follows from the fact that the equation in the definition of $\mathcal{M}(c, \lambda, \mathbf{d})$ has at most $(s-1) k+d_{s}-1$ solutions $\alpha$, and that for every such solution $\alpha$, all $k$ simple roots of the defining primitive polynomial $f$ of $\alpha$ satisfy the equation.

Therefore, by proceeding quite analogously to the proof of Theorem 1 , and since $|\mathcal{Q}|=\phi\left(p^{k}-1\right) / k$, letting $\mathcal{M}(c)$ be the set of $f \in \mathcal{Q}$ such that there exist $d_{1}, \ldots, d_{s}>0$ with $d_{1}+\ldots+d_{s}=k-c$ and with $\alpha^{0}, \ldots, \alpha^{d_{1}-1}, \alpha^{k}, \ldots$ $\ldots, \alpha^{k+d_{2}-1}, \ldots, \alpha^{(s-1) k}, \ldots, \alpha^{(s-1) k+d_{s}-1}$ linearly dependent over $F_{p}$, we 
have

$$
|\mathcal{M}(c)| \leq|\mathcal{Q}| \frac{p^{k}}{\phi\left(p^{k}-1\right)} k(s-1) p^{-c}\left(\begin{array}{c}
k-c-1 \\
s-1
\end{array}\right)=:|\mathcal{Q}| R(c) .
$$

Let $\overline{\mathcal{M}}(c):=\mathcal{Q} \backslash \mathcal{M}(c)$. Then $|\overline{\mathcal{M}}(c)| \geq|\mathcal{Q}|(1-R(c))$. Proceeding as in the proof of Theorem 1 we get

$$
\begin{aligned}
\sum & :=\frac{1}{|\overline{\mathcal{M}}(c)|} \sum_{f \in \overline{\mathcal{M}}(c)} D_{T}^{*(s)}(f) \\
& \leq \frac{1}{p^{k}} \sum_{w=0}^{s-1}(p-1)^{w}\left(\begin{array}{c}
k \\
w
\end{array}\right)\left[\frac{p^{k}}{|\overline{\mathcal{M}}(c)|} c(s-1) \frac{p}{p-1}+1\right]=: A(c) .
\end{aligned}
$$

We then easily finish the proof like the proof of Theorem 1 . The $\mathcal{Q}$-result comes from the fact that $x / \phi(x)=\mathcal{O}(\log \log x)$.

(b) The GFSR method. This method is due to Lewis and Payne [1]. Let $p$ be a prime, and let $k \geq 2$ be an integer. For a primitive characteristic polynomial $f$ of degree $k$ over $F_{p}$ we define the sequence $\left(y_{n}\right)_{n=0, \ldots, T-1}$ of period $T=p^{k}-1$ as in the digital multistep method. For $m \geq 2$ we then choose integers $h_{1}, \ldots, h_{m} \geq 0$ and we put

$$
x_{n}:=\sum_{j=1}^{m} y_{n+h_{j}} p^{-j} \quad \text { for } n=0,1, \ldots
$$

This GFSR sequence has period $T$. In the following we again consider the case $m=k$.

It was shown in [2] (see also Theorem 9.17 of [4]) that for given $f$ of degree $k \geq s \geq 2$ and given initial values $y_{0}, \ldots, y_{k-1}$ not all zero (and for $m=k$ ), for the star-discrepancy $D_{T}^{*(s)}\left(h_{1}, \ldots, h_{k}\right)$ of the $s$-dimensional serial set $\mathbf{x}_{n}:=\left(x_{n}, x_{n+1}, \ldots, x_{n+s-1}\right), n=0, \ldots, T-1$, of the corresponding GFSR sequence $\left(x_{n}\right)_{n=0, \ldots, T-1}$ we have on the average

$$
D_{T}^{*(s)}\left(h_{1}, \ldots, h_{k}\right) \leq c(p, s) \frac{(\log T)^{s}}{T}
$$

with an implied constant depending only on $p$ and $s$, where the average is taken over all $H=\left(h_{1}, \ldots, h_{k}\right)$ with $0 \leq h_{j} \leq T-1$ for $1 \leq j \leq k$. Let $\mathcal{H}$ be the system of all such $k$-tuples $H$. Then again for every $\gamma$ with $0<\gamma<1$, the number of $H$ for which $D_{T}^{*(s)}(H)$ satisfies

$$
D_{T}^{*(s)}(H) \leq \frac{1}{1-\gamma} c(s, p) \frac{(\log T)^{s}}{T}
$$

is at least $\gamma|\mathcal{H}|$. The following Theorem 3 is an improvement of this result:

TheOREM 3. For a prime $p$ let $s \geq 2, m=k \geq s$, a primitive polynomial $f$ of degree $k$ over $F_{p}$, and initial values $y_{0}, \ldots, y_{k-1}$, not all zero, be given. 
Let $T:=p^{k}-1$. For fixed $\gamma, 0<\gamma<1$, the number of $H \in \mathcal{H}$ for which the star-discrepancy $D_{T}^{*(s)}(H)$ of the s-dimensional serial set of the GFSR sequence defined by $f, H$ and the initial values satisfies

$$
\begin{aligned}
D_{T}^{*(s)}(B) \leq & \frac{1}{T}+\frac{1}{p^{k}} \sum_{w=0}^{s-1}(p-1)^{w}\left(\begin{array}{c}
k \\
w
\end{array}\right) \\
& \times\left[(s-1)\left(\frac{p}{p-1}\right)^{2} \frac{2}{1-\gamma}{ }_{p} \log k\right. \\
& \left.+\left(\frac{p}{p-1}\right)^{2} \frac{2}{1-\gamma}\left(1+{ }_{p} \log \frac{4}{1-\gamma}\right)+\frac{1+\gamma}{1-\gamma}\right] \\
= & \mathcal{O}\left(\frac{(\log T)^{s-1} \log \log T}{T}\right)
\end{aligned}
$$

is at least $\gamma|\mathcal{H}|$.

Pr o of. Again (see Theorem 9.14 of [4]), $\mathbf{0}, \mathbf{x}_{0}, \ldots, \mathbf{x}_{T-1}$ form a digital net over $F_{p}$ with the matrices $A_{i}(h)$ with rows $\alpha^{i-1+h_{j}}, j=1, \ldots, k, i=$ $1, \ldots, s$ ( $\alpha$ a root of $f$ in $F_{p^{k}}$ ), playing the role of $A_{i}(B)$ and $A_{i}(f)$ in the proofs of Theorems 1 and 2, respectively.

For a non-negative $c$ we define the sets $\mathcal{M}(\lambda, \mathbf{d})$ and $\mathcal{M}(c)$ as in the proofs of the above theorems. The equation in the definition of $\mathcal{M}(\lambda, \mathbf{d})$ is then equivalent to

$$
\sum_{j=1}^{k} \xi_{j} \alpha^{h_{j}}=0 \quad \text { with } \quad \xi_{j}:=\sum_{i=0}^{s-1} \lambda_{d_{1}+\ldots+d_{i}+j} \alpha^{j} .
$$

Since $s \leq k$ and since $\alpha$ is a primitive element in $F_{p^{k}}$, we see that for $\lambda \neq \mathbf{0}$ not all $\xi_{j}$ are zero and therefore (again since $\alpha$ generates $F_{p^{k}}$ and since $0 \leq h_{j} \leq p^{k}-2$ for all $j$ ) we have $|\mathcal{M}(\lambda, \mathbf{d})| \leq T^{k-1}$. Consequently,

$$
|\mathcal{M}(c)| \leq|\mathcal{H}|\left(\begin{array}{c}
k-c-1 \\
s-1
\end{array}\right) p^{k-c} \frac{1}{T}=:|\mathcal{H}| R(c)
$$

and with $\overline{\mathcal{M}}(c):=\mathcal{H} \backslash \mathcal{M}(c)$ we get

$$
\begin{aligned}
\frac{1}{|\overline{\mathcal{M}}(c)|} \sum_{H \in \mathcal{H}} & D_{T}^{*(s)}(H) \\
\leq & \frac{1}{p^{k}} \sum_{w=0}^{s-1}(p-1)^{w}\left(\begin{array}{c}
k \\
w
\end{array}\right)\left[1+\frac{|\mathcal{H}|}{|\mathcal{M}(c)|} \cdot \frac{p}{p-1} \cdot \frac{1}{1-1 / p^{k}} c\right] \\
= & : A(c) .
\end{aligned}
$$

We finish the proof like the proofs of Theorems 1 and 2 . 


\section{References}

[1] T. G. Lewis and W. H. Payne, Generalized feedback shift register pseudorandom number algorithm, J. Assoc. Comput. Mach. 20 (1973), 456-468.

[2] H. Niederreiter, Point sets and sequences with small discrepancy, Monatsh. Math. 104 (1987), 273-337.

[3] - , The serial test for digital k-step pseudorandom numbers, Math. J. Okayama Univ. 30 (1988), 93-119.

[4] - Random Number Generation and Quasi-Monte Carlo Methods, CBMS-NSF Regional Conf. Ser. in Appl. Math. 63, SIAM, Philadelphia, 1992.

[5] - Factorization of polynomials and some linear-algebra problems over finite fields, Linear Algebra Appl. 192 (1993), 301-328.

[6] - , The multiple recursive matrix method for pseudorandom number generation, Finite Fields Appl. 1 (1995), 3-30.

[7] - Improved bounds in the multiple-recursive matrix method for pseudorandom number and vector generation, ibid. 2 (1996), 225-240.

[8] H. Niederreiter and C. P. Xing, Low-discrepancy sequences obtained from algebraic function fields over finite fields, Acta Arith. 72 (1995), 281-298.

[9] - - - Low-discrepancy sequences and global function fields with many rational places, Finite Fields Appl. 2 (1996), 241-273.

[10] - - -, Quasirandom points and global function fields, in: S. Cohen and H. Niederreiter (eds.), Finite Fields and Applications (Glasgow, 1995), London Math. Soc. Lecture Note Ser. 233, Cambridge Univ. Press, Cambridge, 1996, 269-296.

[11] K. F. Roth, On irregularities of distribution, Mathematika 1 (1954), 73-79.

[12] W. M. Schmidt, Irregularities of distribution, VII, Acta Arith. 21 (1972), 45-50.

13] R. C. Tausworthe, Random numbers generated by linear recurrence modulo two, Math. Comp. 19 (1965), 201-209.

Institut für Mathematik

Universität Salzburg

Hellbrunnerstr. 34

A-5020 Salzburg, Austria

E-mail: Gerhard.Larcher@sbg.ac.at

Web: http://www.mat.sbg.ac.at/people/larcher.html

Received on 8.10.1996

and in revised form on 4.4 .1997 